\title{
Staphylococcus cohnii spp urealyticus: relato de caso de um patógeno incomum
}

\author{
Staphylococcus cohnii spp urealyticus: \\ case report on an uncommon pathogen \\ Pedro Alves d' Azevedo ${ }^{1,2,3}$, Ana Lúcia Sousa Antunes ${ }^{3}$, \\ Marinês Dalla Valle Martino ${ }^{4}$ e Antonio Carlos Campos Pignatari ${ }^{1,2}$
}

\begin{abstract}
RESUMO
Staphylococcus coagulase negativos tem surgido como importantes agentes em infecções de pacientes hospitalizados. Neste estudo, relatamos o caso de bacteremia associada a cateter venoso central devido a Staphylococcus cohnii spp urealyticus isolado em hemocultura de um paciente do sexo masculino, 53 anos, internado em hospital geral da Cidade de São Paulo. Discutimos nesse relato a dificuldade em identificar rotineiramente esse microrganismo no Laboratório de Microbiologia Clínica. Staphylococcus cobnii spp urealyticus é um microrganismo encontrado na pele dos seres humanos como parte da microbiota normal, podendo em algumas situações causar sérias infecções em humanos.
\end{abstract}

Palavras-chaves: Staphylococcus cohnii spp urealyticus. Bacteremia. Identificação.

\begin{abstract}
Coagulase-negative Staphylococcus has emerged as an important agent in nosocomial infections. In this study, we report a case of bacteremia associated with a central venous catheter, caused by Staphylococcus cohnii spp urealyticus that was isolated in blood cultures from a 53-year-old male patient who was admitted to a general hospital in the City of São Paulo. We discuss in this report the difficulty in routinely identifying this microorganism in the clinical microbiology laboratory. Staphylococcus cohnii spp urealyticus is a microorganism found in human skin as part of the normal microbiota, and it can cause serious infections in humans, in some situations.
\end{abstract}

Key-words: Staphylococcus cohnii spp urealyticus. Bacteremia. Identification.

Staphylococcus coagulase negativos ( $\mathrm{SCoN}$ ) tem surgido como importantes agentes em infecções de pacientes hospitalizados, principalmente na corrente sanguiínea ${ }^{4}$. Dentre eles, o Staphylococcus cohnii é reconhecido como um microrganismo componente da microbiota normal de humanos e outros primatas, podendo estar envolvido em casos raros de endocardites, pneumonias, infecções do trato urinário, abscessos cerebrais, artrites sépticas, entre outras infecções ${ }^{359}$. Podem ser encontrados também no ambiente hospitalar ${ }^{57}$. É um coco Gram-positivo, catalase positivo, coagulase negativo, aeróbio e quando isolado de infecções em humanos apresenta um perfil de multi-resistência ${ }^{16}$. Esses microrganismos podem constituir reservatórios de genes de resistência em hospitais ${ }^{6}$. Existem atualmente duas subespécies descritas na literatura, o Staphylococcus cohnii spp cohnii e Staphylococcus cohnii spp urealyticus. No laboratório clínico, a diferença entre estas duas subespécies pode ser feita pelo uso de algumas provas bioquímicas como a urease, a fosfatase alcalina, pirrolidonil arilamidase (teste PYR), beta-glicuronidase, fermentação da maltose/manitol/manose $e^{3}$. A análise morfológica das colônias isoladas em cultura pode ser útil para se diferenciar estas subespécies, pois a colônia do Staphylococcus cohnii spp urealyticus costuma apresentar na maioria dos isolamentos um pigmento (bege-amarelado) ${ }^{3}$. Por outro lado, infecções humanas causadas pelo Staphylococcus cohnii raramente têm sido descritas na literatura ${ }^{79}$.

\footnotetext{
1. Laboratório Especial de Microbiologia Clínica, Universidade Federal de São Paulo, São Paulo, SP. 2. Departamento de Microbiologia/Parasitologia, Fundação Universidade Federal de Ciências da Saúde de Porto Alegre, Porto Alegre, RS. 3. Curso de Pós-Graduação em Ciências Médicas, Fundação Universidade Federal de Ciências da Saúde de Porto Alegre, RS. 4. Setor de Microbiologia, Laboratório Clínico do Hospital Albert Einstein, São Paulo, SP.

Endereço para correspondência: Prof Pedro A. d'Azevedo. Deptartamento de Microbiologia/UFCSPA. Rua Sarmento Leite 245/204, Centro, 90050-170 Porto Alegre, RS. Tel: $55513303-8740$

e-mail: pedro_dazevedo@yahoo.com.br

Recebido para publicação em: 06/03/2007

Aceito em: 07/03/2008
} 


\section{RELATO DO CASO}

Paciente de 53 anos de idade, sexo masculino, admitido no Hospital 9 de Julho, hospital geral da Cidade de São Paulo, em 27/06/2005 com diagnóstico de psoríase e erisipela em membros inferiores. 0 paciente foi tratado inicialmente com ceftriaxone e evoluiu com febre e comprometimento importante do estado geral. No $6^{\circ}$ dia de internação, foi inserido um cateter venoso na veia subclávia direita e associado o antimicrobiano ciprofloxacina. No $11^{\circ}$ dia de internação, apresentou hemocultura positiva para Staphylococcus spp coagulase negativo, sendo introduzido o antimicrobiano teicoplanina. 0 paciente evoluiu com persistência da febre e no $20^{\circ}$ dia de internação foi trocado o cateter venoso central sendo que a cultura do mesmo foi positiva para Staphylococcus spp coagulase negativo com mais de 15 unidades formadoras de colônias. Na ocasião, o ecocardiograma não mostrava evidências de endocardite bacteriana, mas 0 ultra-som de abdômen revelava trombose de veia cava inferior. Uma hemocultura colhida no $22^{\circ}$ dia de internação foi positiva para Pseudomonas aeruginosa multi-resistente, inclusive a carbapenêmicos. 0 paciente evoluiu com sepse, progressão da trombose venosa de veia cava inferior e óbito no $25^{\circ}$ dia de internação.

Os isolados das primeiras duas hemoculturas (sistema automatizado BacAlert $^{\circledR}$ ) foram identificados apenas como Staphylococcus spp coagulase negativo. Por terem algumas características fenotípicas incomuns, as duas amostras foram enviadas a dois centros de referência: o Laboratório de Cocos Gram Positivos da Fundação Universidade Federal de Ciências da Saúde de Porto Alegre (UFCSPA), RS, Brasil que utiliza o processo de identificação convencional e o Laboratório Clínico do Hospital Israelita Albert Einstein, São Paulo, SP, Brasil, que rotineiramente, recorre aos sistemas automatizado VITEK ${ }^{\circledR}$ legacy 1 e 2 (bioMérieux).

\section{DISCUSSÃO}

No Laboratório de Cocos Gram Positivos da UFCSPA, a bacterioscopia dos isolados na coloração de Gram evidenciou cocos Gram-positivos isolados e em pequenos grupos, providos de catalase e ausência da coagulação em plasma de coelho. As amostras foram semeadas em agar-sangue de carneiro (Oxoid, Basingstoke, UK) e após a incubação a $35^{\circ} \mathrm{C} / 24$ horas apresentaram colônias lisas, não hemolíticas, opacas, de tonalidade bege-amarelada que se acentuou com $48 \mathrm{~h}$ de observação. Esse pigmento pode ser melhor visualizado através da raspagem das colônias com uma alça de platina ou um swab. A chave para identificação deste microrganismo no Laboratório Clínico pode ser bastante confusa, pois segundo o Manual of Clinical Microbiology (1999), que sugere outros testes complementares, muitas reações como produção de hemólise, o teste do PYR (pirrolidonilarilamidase) e o teste da desferroxamina apresentam resultados variáveis. Testes adicionais foram realizados como a prova da urease (positiva) e da fosfatase alcalina (positiva) para estes isolados. 0 teste de suscetibilidade aos antimicrobianos foi realizado através do método de discodifusão e os isolados apresentaram susceptibilidade à oxacilina (Oxoid, Basingstoke, UK) e concentrações inibitórias mínimas para vancomicina e teicoplanina de 1 e $3 \mu \mathrm{g} / \mathrm{ml}$, respectivamente, por E-test. Devido a dificuldade na identificação através dos métodos convencionais os isolados foram caracterizados pelos sistemas automatizados VITEK ${ }^{\circledR} 1$ e 2 - Gram Positive Identification Card (BioMérieux) seguindo-se a orientação do fabricante. 0 VITEK ${ }^{\circledR}$ legacy 1 com software versão 10,01 e cartão GPI indicou com 53\% Staphylococcus simulans e 34\% Staphylococcus cohnii. Com esses percentuais, o Laboratório deveria lançar mão de outras provas para uma identificação conclusiva. Já o VITEK ${ }^{\circledR} 2$ com versão do software 4,03 e cartão GP identificou os isolados como sendo o Staphylococcus cohnii spp urealyticus com 99\% de probabilidade. A diferença na probabilidade encontrada entre os dois sistemas decorre essencialmente pelo maior número de provas constituintes do VITEK ${ }^{\circledR} 2$. Salientam-se nesse particular os resultados positivos obtidos na alcalinização L-Lactato, na beta-glucoronidase, na beta galactosidase, na descarboxilação da arginina e na fermentação da D-Manose, substratos inexistentes no $\operatorname{VITEK}^{\circledR} 1$.

0 paciente recebeu teicoplanina por duas semanas apesar da suscetibilidade à oxacilina devido à extensão das lesões cutâneas e gravidade do quadro clínico com possibilidade de infecção por Staphylococcus aureus resistente à oxacilina. A infecção foi considerada como relacionada ao cateter venoso central, que foi positivo para Staphylococcus spp coagulase negativo (SCoN) quando da sua retirada, com o mesmo perfil de suscetibilidade aos antimicrobianos que as amostras de hemoculturas. Na evolução clínica foi feito diagnóstico de trombose de veia cava inferior com comprometimento de veia supra-hepática e extensão até a confluência das veias renais e sepse por Pseudomonas aeruginosa. Apesar das medidas terapêuticas em unidade de terapia intensiva o paciente evoluiu a óbito.

A correta identificação de SCoN no Laboratório de Microbiologia Clínica é muito trabalhosa e poucos laboratórios o fazem. No entanto, cada vez mais descrições de casos e ou surtos mostram que cada espécie tem um papel importante na epidemiologia hospitalar, bem como um perfil diferenciado quanto à resistência aos antimicrobianos. Staphylococcus cobnii spp urealyticus é um patógeno incomum em infecções humanas e a sua correta identificação com testes de suscetibilidade a antimicrobianos pode auxiliar no rumo da terapêutica a ser tomada em cada caso.

\section{REFERÊNCIAS}

1. Drozenova J, Petras P. Characteristics of coagulase-negative staphylococci isolated from hemocultures. Epidemiologie Mikrobiologie Imunologie 49: 51-58, 2000.

2. Kloos WE, Bannerman TL. Update on clinical significance of coagulase-negative staphylococci. Clinical Microbiology Reviews 7: 117-140, 1994.

3. Kloos WE, Bannerman TL Staphylococcus and Micrococcus. In: Murray P, Baron E, Pfaller M, Tenover F, Yolken R (eds) Manual of Clinical Microbiology. American Society for Microbiology, Washington, DC, p. 264-282, 1999. 
4. Marshall SA, Wilke WW, Pfaller MA, Jones RN. Staphylococcus aureus and coagulase-negative staphylococci from blood stream infections: frequency of occurrence, antimicrobial susceptibility, and molecular ( $m e c A)$ characterization of oxacillin resistance in the SCOPE Program. Diagnostic Microbiology and Infectious Disease 30: 205-214, 1998.

5. Szarapinska-Kwaszewska J, Farkas LI. Synthesis of siderophores by strains of Staphylococcus cohnii isolated from various environments. Acta Microbiologica Polonica 52: 261-269, 2003.

6. Szewczyk EM, Rozalskz M. Staphylococcus cohnii-resident of hospital environment: cell-surface features and resistance to antibiotics. Acta Microbiologica Polonica 49: 121-133, 2000.
7. Szewczyk EM, Rozalskz M, Cieslikowski T, Nowak T. Plasmids of Staphylococcus cohnii isolated from the intensive-care unit. Folia Microbiologica 49: 123-131, 2004.

8. Waldon E, Szewczyk EM. Ability of Staphylococcus cohnii strains to adhere to epithelial cells and solid surfaces in the hospital environment. Medycyna Doswiadczalna I Mikrobiologia 54: 109-118, 2002.

9. Yamashita S, Yonemura K, Sumimoto R, Tokunaga M, Uchino M. Staphylococcus cohnii as a cause of multiple brain abscesses in Weber-Christian disease. Journal of the Neurological Sciences 15: 97-100, 2005. 